\title{
(Mostly) dormant comets in the NEO population and the meteoroid streams that they crumble into
}

\author{
Peter Jenniskens \\ SETI Institute, 515 N. Whisman Road, Mountain View, CA 94043 \\ email: pjenniskens@mail.arc.nasa.gov
}

\begin{abstract}
Many of our annual showers do not have active parent comets. In recent years, minor planets have been identified that move among the meteoroids streams. Some streams, such as the Quadrantids, Geminids, and Sextantids, are in such unusual orbits that the probability of a chance association is only of order 1 in $10^{6}$. The streams identify those objects as dormant comet nuclei. Other streams, such as the Phoenicids and $\alpha$-Capricornids are associated with minor planets that were found to be weakly active at their last perihelion passage. All the streams investigated so far are young, less than 2000 years old, and can not have been created in the classical sense of meteoroids being ejected from the comet nucleus by water vapor drag. Instead, these (mostly) dormant comets lost fragments at some point in the past, which crumbled into meteoroid streams. Scars of such events are now identified on the surface of active Jupiter family comets $9 \mathrm{P} /$ Tempel 1 and $81 \mathrm{P} /$ Wild 2. Thus, the meteor showers on Earth bear witness to what is the dominant mass-loss mechanism of comets in the inner solar system, a process that can account for much of the zodiacal cloud dust and the zodiacal dust bands.
\end{abstract}

Keywords. Meteors; meteoroids; meteor showers; interplanetary medium; comet mass loss; comet fragmentation

\section{Introduction}

When Whipple (1951) identified the drag of water vapor as a potent force to accelerating grains off the surface of a comet, his mathematical description of dust ejection by water vapor drag first described the formation of a meteoroid stream. After one orbit, the meteoroids return at a different time, spreading along the comet orbit into a comet dust trail. We now know that the meteor outbursts of some long-period comets are understood as being caused by the 1-revolution dust trails that wander occasionally in Earth's path. The 2-revolution trail is usually not detected because perturbations of the orbital period cause trails to fold and break and disperse in nodes. The orbital period of the dust of Halley-type comets is less changed, so that dust trails can persist for several revolutions, leading to multiple meteor storms when Earth travels through the debris field of a Halley-type comet. The dust of Jupiter-family comets is strongly perturbed when Jupiter meets the dust at aphelion, resulting in rapid distortions as a function of time of ejection. This changes the dust trail cross section and results in rapid spreading of the dust along the comet orbit. Some trail segments that avoid close encounters escape as intact trailets that can cause meteor outbursts on Earth (e.g., Jenniskens 2006a).

A problem with this scenario is that the majority of our meteor showers have no known parent body. As of January 2003, these included the major showers of Quadrantids, Geminids, Daytime Arietids, Taurids, and Sextantids, as well as the well-known $\alpha$ Capricornids, $\delta$-Aquariids, and Phoenicids. These are Jupiter-family comets and streams with a shorter orbital period that are now mostly decoupled from Jupiter. It was surmised, 
that the parent bodies of these streams had now completely disintegrated, or perhaps had evolved into orbits quite different from the meteoroids that we now see on Earth as a meteor shower. However, many of these streams, such as those responsible for the Quadrantids, the Geminids, the $\alpha$-Capricornids and the Sextantid meteor shower, are so little dispersed that they can not be older than 2000 years.

In the past three years, all of these streams were found to have associated minor planets. $2003 \mathrm{EH}_{1}$ moves in the highly inclined Quadrantid stream, $2002 \mathrm{EX}_{12}$ moves among the $\alpha$-Capricornids (and was weakly active when at perihelion), $2004 \mathrm{TG}_{10}$ is a better match to the Northern Taurids than comet 2P/Encke, and 2005 UD moves among the small- $q$ Daytime Sextantids (Jenniskens 2006a). Also, $2003 \mathrm{WY}_{25}$ is a fragment of comet D/Blanpain responsible for the Phoenicid outburst of 1956, and the Daytime Arietids are in the same orbit as the Marsden Sungrazer group of comet fragments. This leaves no further doubt that (3200) Phaethon is also the parent of the Geminid shower.

These streams appear to originate in discrete formation events, presumably a form of fragmentation. In some cases, the single-year sighting of a comet may mark that event. In each case, the amount of mass in the stream is less or equal to the largest remaining fragment discovered so far. Instead of a catastrophic fragmentation, these streams appear to be the result of the spill-off of a comet fragment, which subsequently broke into meteoroids. The scars of this spill-off have now been identified on active short-period comet $9 \mathrm{P} /$ Tempel 1.

\section{Individual meteor shower - parent body associations}

\subsection{The Geminids and (3200) Phaethon}

Meteor showers were associated to minor planets ever since Whipple (1983) noticed that 1983 TB Phaethon moved among the very short-period $(P \approx 1.59 \mathrm{yr})$ and eccentric Geminid meteoroids. However, in no other case was the agreement between meteoroid stream and proposed parent body as good. Even more, the Geminid-Phaethon association was long in doubt.

The Geminids have an unusually low perihelion distance $(q \approx 0.14$ AU), and both meteoroids and minor planet surface have been sintered in the heat of the Sun, which can warm a dust grain to above $700^{\circ} \mathrm{K}$. Probably because of that, this minor planet resembles a B-type asteroid with a high 0.11 geometric albedo, much higher than that of known dormant comet nuclei, and the meteoroids penetrate deeper in the atmosphere than known cometary meteoroids (albeit not as deep as known asteroidal meteoroids). Many have assumed that Phaethon is an asteroid that had evolved independently in a similar orbit as the Geminids, despite the low probability for doing so $\left(\approx 1\right.$ in $2 \times 10^{6}$, depending on the actual number of objects in this sparsely sampled population and the extent of the stream).

It has since been shown that the Geminids appear to have been created close to perihelion, where the probability of an asteroidal collision is low (Gustafson 1989).

\subsection{The Quadrantids and $2003 \mathrm{EH}_{1}$}

A second example was identified only twenty years later (Jenniskens 2003). In October 2003, the parent body of the Quadrantid shower was identified among newly discovered minor planets (Table 1). The mean orbit of the Quadrantids in Table 1 is that derived from photographic observations by the Dutch Meteor Society (Jenniskens et al. 1997). Due to the steep $72^{\circ}$ inclination of the orbit, the probability of a chance association with unrelated minor planets is also only about 1 in $2 \times 10^{6}$. This object, $2003 \mathrm{EH}_{1}$, passed Earth's orbit in excess of $0.2 \mathrm{AU}$, and was therefore not immediately recognized 
as a near-Earth object. However, because the aphelion of the orbit is so close to that of Jupiter, the perihelion distance of the object (and of that of the Quadrantid meteoroids) is strongly perturbed, evolving in and out of Earth's orbit. It was shown that 2003 $\mathrm{EH}_{1}$ was perturbed only very recently into an orbit on the outer parts of the stream (Jenniskens 2004).

Table 1. Comparison of orbital elements of Quadrantids and proposed parent bodies (angles referred to J2000).

\begin{tabular}{l|lllllll}
\hline Name & Epoch & $a(\mathrm{AU})$ & $q(\mathrm{AU})$ & $i\left(^{\circ}\right)$ & $\omega\left(^{\circ}\right)$ & $\Omega\left(^{\circ}\right)$ & $\varpi\left(^{\circ}\right)$ \\
Quadrantids & 1995 & 3.14 & 0.979 & 71.2 & 172.0 & 283.3 & 95.3 \\
$2003 \mathrm{EH}_{1}$ & 2003 & 3.126 & 1.193 & 70.79 & 171.37 & 282.94 & 94.3 \\
\hline $2003 \mathrm{EH}_{1}$ & 1490 & 3.10 & 0.580 & 65.7 & 163.7 & 286.5 & 90.2 \\
$\mathrm{C} / 1490 \mathrm{Y}_{1}$ & 1490 & $\infty$ & 0.761 & 73.4 & 164.9 & 280.2 & 85.1 \\
\hline
\end{tabular}

Until that discovery, the Quadrantid shower was thought to originate from comet 96P/Machholz 1, now in a very different orbit, with the meteoroids having evolved at a different pace along a secular cycle than the parent comet (presumably in a slightly shorter orbital period). An age of the stream of about 4000 years was implied. However, new precise photographic observations of Quadrantid meteoroids showed that the meteoroids moved in very similar orbits, passing just inside of Jupiter's orbit. That implied that the stream could not be older than about 500 years (Jenniskens et al. 1997). The Quadrantids are a massive stream, which can not have been created in normal Whippletype ejection by water vapor drag in the short time available. A breakup is implied.

The moment of breakup may have been observed by Chinese astronomers in early 1491, when a comet was observed in a plane similar to that of the Quadrantids. Due to frequent close encounters with Jupiter, it is not easy to link $2003 \mathrm{EH}_{1}$ to the sightings of C/1490 Y $12003 \mathrm{EH}_{1}$ was recovered at ESO/NTT on December 24, 2003 (E. Jehin, M. Billeres), resulting in a better defined orbit. Using the Orbfit program by Andrea Milani and coworkers, Giovanni B. Valsecchi, Jeremie Vaubaillon, and the author identified two possible solutions at less than $3 \sigma$ from the nominal orbital solution. A more precise orbit of $2003 \mathrm{EH}_{1}$ is needed before it can be established that this comet lost a fragment in or just before 1490 and at that time created the Quadrantid stream.

\subsection{The Phoenicids and $2003 W Y_{25}$}

A third case was identified a year later when minor planet $2003 \mathrm{WY}_{25}$ was discovered to be a fragment of comet D/1819 $\mathrm{W}_{1}$ Blanpain (Jenniskens \& Lyytinen 2005). That comet was observed only once in 1819 and the orbit is uncertain. After an initial similarity in angular elements of order $17^{\circ}$, it was found that that similarity had improved to only $0.2^{\circ}$ after the comet orbit was observed for a more extended period of time (Jenniskens \& Lyytinen 2005). Subsequently, Jewitt (2006) discovered from past images taken on March 10, 2004, that $2003 \mathrm{WY}_{25}$ was at that time an active comet, the smallest known comet nucleus at this time, creating dust at a rate of only $0.01 \mathrm{~kg} / \mathrm{yr}$. At this rate, this comet fragment could not have generated the Phoenicid meteoroid stream. Blanpain was so active in 1819 that $2003 \mathrm{WY}_{25}$ is probably much smaller than Blanpain. This can be a fragment from a breakup that most likely occurred during or just before 1819 .

An outburst of Phoenicids was observed in 1956. Jenniskens \& Lyytinen (2005) investigated whether this breakup could have occurred at or just before 1819, with Blanpain being the manifestation of the immediate aftermath. After modeling the meteoroids 
released during a fragmentation event in 1819, we were able to demonstrate that the dust would have moved in Earth's path in 1951 and 1956, but not in other years since.

\subsection{The $\alpha$-Capricornids and 2002 $E X_{12}$}

Next, Paul Wiegert (University of Western Ontario) and the author independently found that $2002 \mathrm{EX}_{12}$ moved in an orbit similar to that of the $\alpha$-Capricornids, albeit slightly further evolved along the secular cycle. This object, too, was discovered to be a comet in July of 2005, and is now known as 169P/NEAT. The $\alpha$-Capricornids have a very dispersed period of activity due to the low inclination of the orbit, but the radiant dispersion in perpendicular direction is very small.

\subsection{The DaytimeArietids and the Marsden Sunskirter comet fragments}

In the mean time, a similarity had been observed between the orbit of the Daytime Arietids and the comet fragments of the Marsden group of sungrazers by David Seargent (Kracht et al. 2002). The latter have a slightly smaller perihelion distance, but the orbit passes very close to Earth orbit. Due to the limited viewing area of the SOHO satellite, and the short activity curve of these small comet fragments, the orbital period of the Marsden sungrazer group could not be determined. In 2004, it was found that some fragments appeared to return after $\sim 5.5$ years (Marsden 2004), making this a shortperiod comet, making it more than likely that whatever was responsible for this large family of comet fragments is also responsible for the Daytime Arietids, even though this stream has a relatively short $P=1.6-2.3$ yr orbital period.

The Marsden and Kracht group of sunskirters and comet Machholz, as well as the Daytime Arietids and $\delta$-Aquariid showers are all part of a complex (the Machholz Complex) of objects (perhaps including the Quadrantid parent $2003 \mathrm{EH}_{1}$ ) that evolved along the same secular cycle. Recently, Sekanina \& Chodas (2005) identified a mechanism for rapid dispersion along this cycle, by invoking an initial fragmentation of the progenitor body followed by a close encounter of the fragment stream with Jupiter in AD 1059. This puts the formation age of the Marsden group of fragments after 1059 AD.

The related $\delta$-Aquariids may be associated with the Kracht group, or with a new yet undiscovered group of comet fragments.

\subsection{The Taurids}

Another such group of comet fragments is the Taurid Complex. It was originally proposed based on minor planets linked to Taurid meteoroid orbits by means of the $D$-criterion. In this manner a large number of associated bodies were found by Clube, Napier, and others, that implied a huge $43 \mathrm{~km}$ diameter parent broken up about 20000 years ago (Clube \& Napier 1984). Comet fragments hitting the Earth were proposed as the cause of climate and environmental changes on Earth. Since, it has been found that all of the originally proposed objects (outside of $2 \mathrm{P} /$ Encke) have asteroidal colors ( $\mathrm{S}$ or $\mathrm{O}$ type taxonomy) and, therefore, in all likelihood are unrelated to the Taurid shower. All those objects have $q>0.4$ AU (mostly with $q \approx 0.55 \mathrm{AU}$ ), larger than the Taurid meteoroids with $q \approx 0.35$ AU.

Nevertheless, the large nodal extend of the Taurid stream (September - December) and the presence of southern and northern branches implies that the meteoroids evolved at least one full cycle of about 5000 years, and possible multiple cycles. In contrast, clusters of orbits in the meteoroid stream and the general "fresh" appearance (numerous flares) of the meteors suggest that some Taurids were only fairly recently released. 
Comet $2 \mathrm{P} /$ Encke itself was never a good fit to the Taurids observed on Earth. The comet has evolved further along the secular cycle, now with a relatively high inclination of $12^{\circ}$ and a node at Mercury's orbit.

Only very recently, a parent body was identified with $q$ and other orbital elements similar to that of the northern Taurids: $2004 \mathrm{TG}_{10}$ (Jenniskens 2006b). Next to comet $2 \mathrm{P} /$ Encke, this may be the second fragment of an original larger body. That body was perhaps as large as comet $1 \mathrm{P} /$ Halley, but certainly not as large as the $43 \mathrm{~km}$ sized parent body proposed by Clube and Napier.

\subsection{The Geminids and Sextantids}

It is now clear that also the Geminids originate from (3200) Phaethon. Phaethon and its stream are also part of a larger complex of objects. A second meteor shower is known with similar orbital elements, but at a different position along the secular cycle. That is the Daytime Sextantid shower. Again, it was unlikely that the Sextantids evolved from the same fragmentation event as that responsible for the Geminids. The Geminids are not old enough to account for the difference in orbital elements.

Recently, Ohtsuka (2005) identified 2005 UD as the likely body responsible for the Sextantid meteoroid stream in a separate fragmentation event. It is likely that other minor planets exist that are part of this same Phaethon Complex.

Table 2. Meteor showers and their parent comets. Orbital elements, Tisserand parameter, Nuclear magnitude, and Formation epoch.

\begin{tabular}{|c|c|c|c|c|c|c|c|c|c|}
\hline Shower/comet & Epoch & $a(\mathrm{AU})$ & $q(\mathrm{AU})$ & $\mathrm{i}$ & $\omega$ & $\Omega$ & $T_{J}$ & $H N$ & Formation \\
\hline Tau-Herc & \multicolumn{9}{|c|}{ source of future showers } \\
\hline 73P/S.-W. 3 & $(1995)$ & 3.06 & 0.933 & 11.4 & 198.8 & 69.9 & +2.78 & +17.7 & AD 1995 \\
\hline \multirow{2}{*}{$\begin{array}{l}\text { Andromedids } \\
\text { 3D/Biela }\end{array}$} & & 2.90 & 0.777 & 7.5 & 242.7 & 225.5 & & & \\
\hline & $(2004)$ & 3.49 & 0.798 & 7.5 & 236.2 & 213.8 & +0.78 & $>+7.1$ & AD 1840 \\
\hline \multirow{2}{*}{$\begin{array}{l}\text { Phoenicids } \\
2003 \mathrm{WY}_{25}\end{array}$} & & $(3.05)$ & 0.985 & 15.9 & 358.2 & 74.1 & & & \\
\hline & $(1956)$ & 3.07 & 0.991 & 9.6 & 360.1 & 74.4 & +0.51 & $>+8.5$ & $\approx \mathrm{AD} 1819$ \\
\hline \multirow{2}{*}{$\begin{array}{l}\text { Quadrantids } \\
2003 \mathrm{EH}_{1}\end{array}$} & & 3.14 & 0.979 & 72.0 & 172.0 & 283.3 & & & \\
\hline & $(2006)$ & 3.13 & 0.979 & 70.8 & 171.4 & 283.0 & +3.89 & +17.7 & $\approx \mathrm{AD} 1490$ \\
\hline \multirow{2}{*}{$\begin{array}{l}\text { Daytime Arietids } \\
\text { Marsden-group }\end{array}$} & & 1.75 & 0.094 & 27.9 & 29.5 & 78.7 & & & \\
\hline & $(2004)$ & 3.33 & 0.0483 & 26.8 & 23.2 & 81.5 & $\approx+1.8$ & $>+18$ & $\geqslant$ AD 1059 \\
\hline \multirow{2}{*}{$\begin{array}{l}\text { Geminids } \\
(3200) \text { Phaethon }\end{array}$} & & 1.37 & 0.141 & 24.0 & 324.4 & 261.5 & & & \\
\hline & $(2005)$ & 1.27 & 0.140 & 24.2 & 325.3 & 262.5 & +4.51 & +14.6 & $\approx \mathrm{AD} 1030$ \\
\hline \multirow{2}{*}{$\begin{array}{l}\text { Northern Taurids } \\
2004 \mathrm{TG}_{10}\end{array}$} & & 2.12 & 0.350 & 3.1 & 294.9 & 226.2 & & & \\
\hline & $(2005)$ & 2.24 & 0.315 & 3.6 & 298.4 & 223.9 & +2.99 & +19.5 & $\approx \mathrm{AD} 600$ \\
\hline \multirow{2}{*}{$\begin{array}{l}\text { Capricornids } \\
2002 \mathrm{EX}_{12}\end{array}$} & & 2.62 & 0.602 & 7.7 & 266.7 & 128.9 & & & \\
\hline & $(2005)$ & 2.60 & 0.605 & 7.6 & 266.0 & 128.8 & +2.89 & +26.5 & $\approx \mathrm{AD} 10$ \\
\hline
\end{tabular}

\subsection{Andromedids and $\tau$-Herculids}

The idea that the fragmentation of comets is a source of meteoroids causing meteor showers on Earth was first proposed following the 1872 and 1885 Andromedid storms, which occurred after the breakup of lost comet 3D/Biela in 1840, and the continued fragmentation of the comet observed in the returns of 1846 and 1852. It is not certain, however, that the breakup added much to the normal dust ejection of this active comet. 


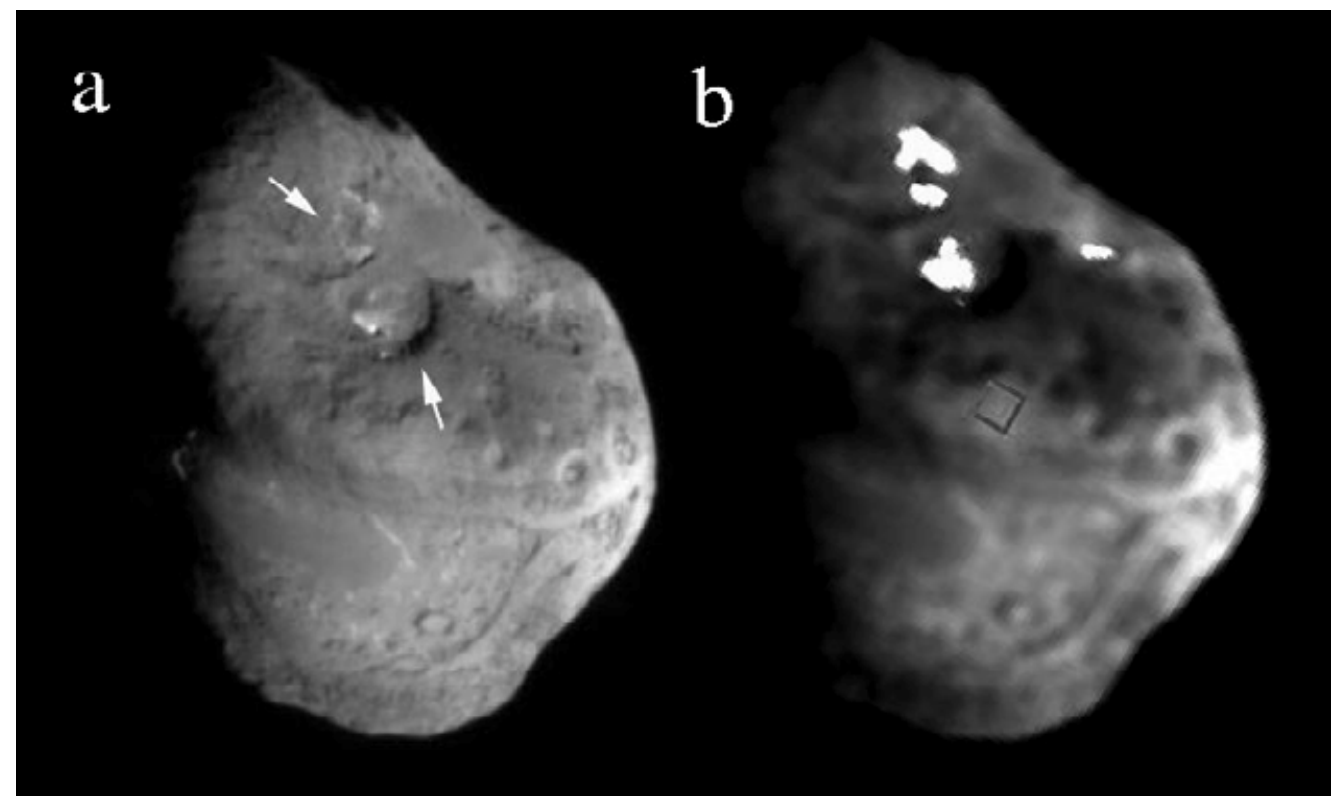

Figure 1. Deep Impact target 9P/Tempel 1. (a) Arrows mark areas that might be the scars from recent fragmentation. (b) White areas are where water ice was detected very near the surface. Photo: NASA/JPL/Deep Impact.

Although the meteor showers were spectacular, they were not more intense than the more recent Draconid storms of 1933 and 1946, thought to have been caused by comet 21P/Giacobini-Zinner through normal Whipple-type comet dust ejection through water vapor drag. Even though the Andromedid storms can be traced to dust trails in the Earth's path dating from 1846 and 1852, when the comet was in breakup, there were no dust trail encounters in other years that could have proven that normal activity of 3D/Biela wasn't capable of creating similar showers.

Another shower created during the breakup of an active comet is that of $73 \mathrm{P} /$ Schwassmann-Wachmann 3. This relatively young comet is still ice-laden and the fragmentation may prove helpful in understanding what underlies the breakup of other comets. The meteoroids from the 1995 breakup will be near Earth in 2022 .

\section{The scars of fragmentation}

Active Jupiter Family Comets should show the scars of these fragmentation events. Ten days after the impact of Deep Impact on 9P/Tempel 1, I identified two circular features as the potential scars of cometesimal spill-off (from Jenniskens 2006b: Fig. 3a). Interestingly, it was subsequently reported that Deep Impact had detected water ice near the surface at these exact locations (A'Hearn et al. 2005). Water ice must be present relatively low below the surface, so that water vapor can seep to the surface and freeze there. It appears that relatively recently two (or three) $0.5-\mathrm{km}$ sized fragments broke from the comet, creating a meteoroid stream in the process. These fragments must have been relatively pancake shaped, perhaps as a result of the accumulation process.

Indeed, fragmentation can explain morphological features on other comets as well. Pits and shallow depressions are common on $81 \mathrm{P} /$ Wild 2, all of which are more likely due to the loss of cometesimals than due to impacts. The morphology of those pits is 
very different than that of the impact craters on the Saturnian moon Phoebe, which is presumably a captured comet because of its retrograde motion.

\section{Conclusions and interpretations}

I conclude that our main showers are relatively young, less than 2000 years old. The discrete fragmentation through the spill-off of boulders, loss of cometesimals, or catastrophic disruption is a common phenomenon among the population of dormant comet nuclei in the inner solar system. It is, in fact, their main mass-loss mechanism.

Many more ecliptic meteoroid streams have now associated (mostly) dormant comets that are still in very similar orbits. The ones listed in Table 2 are only the most well established associations. Many others are listed in Table 7 in the book "Meteor Showers and their Parent Comets" (Jenniskens 2006a). Most of these concern streams in low-inclination or less eccentric orbits, with much higher likelihood of random asteroids wandering among the meteoroids. Each of these cases needs further study.

From the mass of the streams and that of the remaining fragments, it appears that the streams typically represent an amount of mass similar to that of the mass of the remaining fragment. If each breakup adds about 1000 billion $\mathrm{kg}$ of dust, then a steady state of about 300000 fragmentation events over the course of 20000 years could account for the current mass of dust in the zodiacal cloud (e.g., Hughes 1993). That amounts to 15 fragmentation events per year over the whole cloud of comets in all forms in the inner solar system. To produce our about 105 known antihelion streams (Jenniskens 2006a), this would demand that a fraction of $105 / 300000=0.04 \%$ of those breakups evolved dust into Earth's path if the streams survive (at high enough dust density) for 20000 years, or $0.4 \%$ if the streams survive for only 2000 years. Those are reasonable numbers.

This young age of the zodiacal cloud argues against the asteroid families being the source of the zodiacal dust bands. In fact, the breakup of (mostly) dormant comets in the inner solar system is a much more likely mechanism. The argument that there are no known active Jupiter family comets in a suitable orbit does not longer apply. There is a wealth of potentially responsible minor bodies that are now dormant.

\section{Acknowledgements}

Jeremie Vaubaillon and Esko Lyytinen contributed to this work by numerical modeling the dynamics and evolution of meteoroid streams. PJ is supported by NASA's Planetary Astronomy and Planetary Atmospheres programs.

\section{References}

A'Hearn, M.F., Belton, M.J.S., Delamere, W.A., Kissel, J., Klaasen, K.P., McFadden, L.A., Meech, K.J., Melosh, H.J., Schultz, P.H., Sunshine, J.M., Thomas, P.C., Veverka, J., Yeomans, D.K., Baca, M.W., Busko, I., Crockett, C.J., Collins, S.M., Desnoyer, M., Eberhardy, C.A., Ernst, C.M., Farnham, T.L., Feaga, L., Groussin, O., Hampton, D., Ipatov, S.I., Li, J.-Y., Lindler, D., Lisse, C.M., Mastrodemos, N., Owen, W.M., Richardson, J.E., Wellnitz, D.D. \& White, R.L. 2005, Science 310, 258

Clube, S.V.M. \& Napier, W.M. 1984, Mon. Not. R. Astron. Soc. 211, 953

Gustafson, B.A.S. 1989, Astron. Astrophys. 225, 533

Hughes, D.W. 1993, in: J. Stohl \& I.P. Williams (eds.), Meteoroids and their Parent Bodies (Bratislava: Astron. Inst. Slovak Acad. Sci.), p. 15

Jenniskens, P. 2003, IAUC 8252

Jenniskens, P., Betlem, H., de Lignie, M., Langbroek, M. \& van Vliet, M. 1997, Astron. Astrophys. 327,1242

Jenniskens, P. 2004, Astron. J. 127, 3018 
Jenniskens, P. \& Lyytinen, E. 2005, Astron. J. 130, 1286

Jenniskens, P. 2006a, Meteor Showers and their Parent Comets (Cambridge: Cambridge University Press)

Jenniskens, P. 2006b, in: A. Graps (ed.), Proceedings of the "Dust in Planetary Systems" workshop, Kuaui, Hawaii

Jewitt, D. 2006, Astron. J. 131, 2327

Kracht, R., Meyer, M., Hammer, D., Marsden, B.G. \& Seargent, D.A.J. 2002, MPEC 2002-E25

Marsden, B.G. 2004, MPEC 2004-X73

Ohtsuka, K., 2005 CBET 283

Sekanina, Z. \& Chodas, P.W. 2005, Astron. J. Suppl. Ser. 161, 551

Whipple, F.L. 1951, Astrophys. J. 113, 464

Whipple, F.L. 1983, IAUC 3881, 1 